\title{
Detecting vortices within unsteady flows when using single-shot PIV
}

\author{
C. E. Simpson ${ }^{1} \cdot$ H. Babinsky² $\cdot$ J. K. Harvey ${ }^{2}$ (I) S. Corkery ${ }^{2}$
}

Received: 15 August 2017 / Revised: 15 May 2018 / Accepted: 16 May 2018 / Published online: 7 July 2018

(c) The Author(s) 2018

\begin{abstract}
This paper address problems that arise when investigating vortices within an unsteady flow when a true picture of the velocity field can only be obtained from single-shot PIV surveys (SS-PIV). The implications of noise within data are considered when it is not legitimate to minimise this by averaging the results from repeated tests. An improvement to be applied when there are nearby vortices to the non-localised vortex detection scheme proposed by Graftieaux et al. (Meas Sci Technol 12:1422-1429, 2001) is presented. This specifically acknowledges the gradients in the background shear due to velocities induced by these neighbours. The errors that arise in estimating the vortex strength and core radius when the laser plane is not perpendicular to the vortex axis are also discussed. The averaging of SS-PIV data from survey planes that are closely separated either in time or space is proposed as a way to improve the signal-to-noise ratio for SS-PIV surveys. Sample results for the flow associated with the front wing-wheel combination of a F1 car are presented to illustrate the effectiveness of the techniques proposed. In this example the wing is located just ahead of the wheel which has a very unsteady wake. Thus, the vortices shed from the wing are perturbed in a random fashion as they interact with this wake, necessitating the use of SS-PIV. The results demonstrate that the techniques proposed here are capable of identifying the vortices and can determine their properties adequately.
\end{abstract}

\section{Introduction}

Vortices play a decisive role in determining the structure of very many flows and the advent of particle image velocimetry (PIV) has provided experimentalists with a powerful and versatile tool with which to study them. The 'wavelet' approach, based on seeking a correlation between the PIV measurements and some idealised vortex representation, for example, the Lamb-Oseen (L-O) model (Lamb 1993) has proved to be very effective for this purpose [see, for example, Schramm et al. (2004), Varun et al. (2008) and Wang et al. (2015)]. The success of this depends in part on how closely the measured data approximates to the idealised vortex, but also on how accurately they have been acquired. The errors that occur if PIV is used to acquire the data can be classified into two groups: random errors and systemic or bias errors. The random errors arise from a variety of

$\triangle$ J. K. Harvey

jkh28@cam.ac.uk

1 Present Address: Mercedes-AMG Petronas Motorsport, Brackley, UK

2 Department of Engineering, University of Cambridge, Cambridge, UK sources. Instrumentation noise is a significant contributor and has its origins within the optical system (photon noise generated by the light falling on the camera sensor) and from electronic sources (from the camera sensor and from its associated electronics). Random discretisation errors also arise in processing the information digitally and from uncertainties in determining the particle positions using subpixel interpolation algorithms. The noise has the effect of reducing the relative height and the position of the image correlation peak and leads to uncertainties in evaluating the value of the velocities. It is common practice to minimise the random errors using statistical methods by repeating a test several times and averaging the results. However, this is only legitimate if the flow is strictly steady or repeatable. If features in the flow move from one survey to the next the averaging process smears the results. Conditional averaging can be attempted to mitigate this, but the steep velocity gradients within vortices render them particularly susceptible to this type of error even if the movement is only slight. The averaging leads to an apparent reduction in their measured strength, an over estimate of core diameters as well as errors in determining their location. For flows where the vortex position varies with time, a true picture can only be obtained 
from single-shot PIV surveys (SS-PIV) which means tolerating an inevitably inferior signal-to-noise ratio.

This paper addresses three issues that arise from using SS-PIV to detect vortices in complex flows. First, the effectiveness of vortex detection schemes in dealing with noisy data is discussed. Then, two further issues are considered that are of particular relevance to the flow that is the focus of this paper. This involves a cluster of trailing vortices shed from a wing that spiral around each other in close proximity. Each vortex is thus subjected to a spatially varying induced velocity due to its neighbours. The widely used Graftieaux vortex location algorithm (Graftieaux et al. 2001) is based on the assumption of a superimposed uniform external shear acting on each vortex. This assumption is questioned and an improved scheme that acknowledges the variations in shear due to the nearby vortices is put forward. The third issue that is considered arises because of the errors incurred in characterising a vortex when its axis is inclined to the PIV laser sheet, for instance, when studying a cluster of spiralling vortices for which it is only possible at best to arrange for one of them to have its axis perpendicular the laser sheet.

\section{Vortex tracking and characterisation with SS-PIV}

There are a variety of schemes that can be used to locate vortices. These fall into two distinct groups; either they are the so called local methods [see, for example, Chakraborty et al. (2005)], based on critical point theory that considers the topology of flow at that point, or they are non-localised schemes that concentrate on the topology of all of the flow within an area that is comparable to the size of the vortex. The latter identify regions of swirling flow from the cluster of velocity vectors that fall within this area.

The first group, although still widely used to analyse computed flows, are now less frequently employed for processing PIV data because of their sensitivity to signal noise. In contrast, non-local schemes have proved to be very successful in identifying coherent vortical structures even when these are embedded within significant levels of turbulence [see Jiang et al. (2002), Pemberton et al. (2002) and Vollmers (2001)]. Graftieaux et al. defined a now familiar dimensionless scalar vortex detection parameter for the point $P$ given by

$\gamma(P)=\frac{1}{S} \int_{M \in S} \frac{\left(\mathbf{P M} \times\left(\mathbf{u}_{M}-\mathbf{u}_{P}\right)\right) \cdot z}{\mathbf{P M} \cdot \mathbf{u}_{M}-\mathbf{u}_{P}} \mathrm{~d} S=\frac{1}{S} \int_{S} \sin \left(\theta_{M}\right) \mathrm{d} S$,

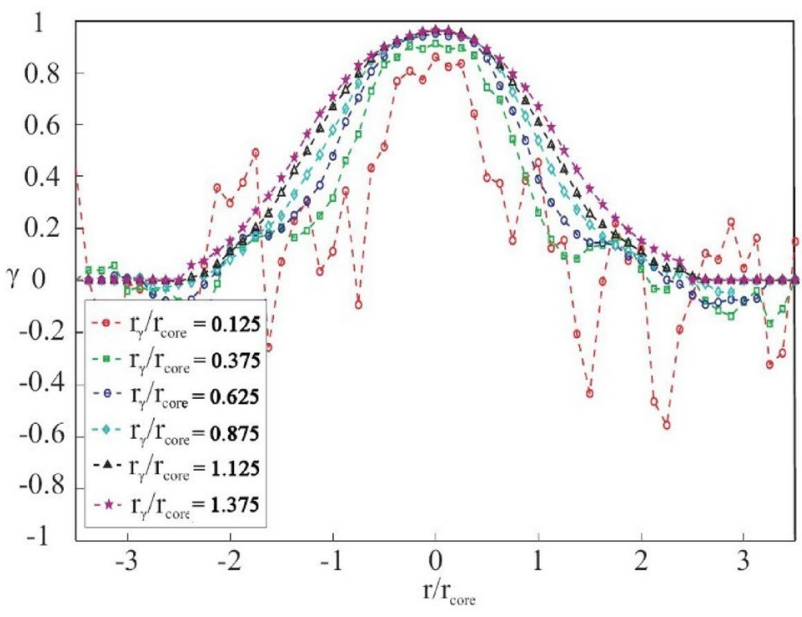

Fig. 1 The $\gamma(P)$ distribution for a Lamb-Oseen vortex with $20 \%$ noise added for various interrogation window sizes

where $S$ is a two-dimensional area surrounding the point $P$ and $M$ is another point that lies within $S .{ }^{1} \mathbf{u}_{\mathrm{p}}$ is the average convection velocity over $S$ given by $\mathbf{u}_{P}=1 / S_{S} \mathbf{u d} S$. (Note that Graftieaux used the upper-case symbol $\Gamma_{2}$ for this parameter, but we have changed this to $\gamma$ to avoid confusion with the circulation). The existence of the vortices can thus be inferred from the peaks in the $\gamma$ profile. However, the location of the vortex centre is not very precise due to the peaks in the $\gamma$ profiles being flat topped (see Fig. 14 in the "Appendix").

To explore the robustness of Graftieaux's scheme in dealing with noise, we evaluate $\gamma$ for a synthesised set of velocity vectors for an isolated $\mathrm{L}-\mathrm{O}$ vortex (details of which are given Appendix). To this, noise is added which is randomly sampled from a normal distribution that has a zero mean velocity and a standard deviation of $20 \%$ of the maximum tangential velocity in the vortex. This is higher than usually experienced in experiments, and certainly more than in the tests to be described later, and thus presents a stringent test of the ability of the method to identify vortical structures from noisy data.

To illustrate the efficiency of the Graftieaux scheme in filtering noise, profiles of $\gamma$ have been obtained using different sized interrogation windows. The results are shown in Fig. 1. A square region has been used, the size being given by the ratio of the window width $r_{\gamma}$ to the core radius $r_{\text {core }}$. When this ratio is small the noise seriously interferes with the profiles. However, as the size increases the effects of

\footnotetext{
1 The function within the integral is equivalent to the sine of $\theta_{M}$, the angle between the position vector $\mathbf{P M}$ and the adjusted velocity $\mathbf{u}_{M}-\mathbf{u}_{P}$ at $M$. We have explored using other functions of $\theta_{M}$ but it appears that the sine function is an optimum choice.
} 
the noise diminish rapidly and by $r_{\gamma} / r_{\text {core }}=1.125$ or 1.375 (in this case corresponding to 324 or 484 sampled vectors) there is sufficient averaging for the curves to very closely approximate to that shown in the "Appendix" for a noise free vortex. The existence of the vortex can thus be inferred reliably from the $\gamma$ profile. A significant finding is the rapidity with which the noise suppression improves as the number of points within the region from which data are taken increases, providing this region is comparable to the core size. The method is thus well suited to identifying vortices from SSPIV data. Further analysis has demonstrated that with this scheme, while it is just possible to detect the presence of this vortex with only a $5 \times 5$ mesh centred on its core, this is insufficient to determine its properties adequately.

The Graftieaux scheme only detects the presence of a vortex and hence the next step in characterising it is the determination of its circulation $\Gamma_{0}$. In this study we do this by seeking the best correlation between the measured velocity field and an idealised L-O vortex. This is thought to be superior to other possible methods as it takes into account all the velocity measurements within the vortex. The alternative of integrating the velocity around a bounding contour is more susceptible to contamination from neighbouring vortices. There is also the possibility of summing the vorticity within the region. However, this suffers from the inherent difficulty of evaluating vorticity accurately from discretised data especially if these contain noise, as it involves differentiation. The correlation method has the additional advantage that it yields an estimate for the size of the vortex in terms of the core radius $r_{\text {core }}$ of the $\mathrm{L}-\mathrm{O}$ vortex and gives a more precise estimate of the vortex centre than is possible from the $\gamma$ distribution.

The correlation scheme used in this study follows that proposed by Bertényi (2001) and Bertényi and Graham (2007) who sought the best fit for the distribution of the tangential velocity $\mathbf{u}_{\theta}(r, \theta)$ to the values for the $\mathrm{L}-\mathrm{O}$ vortex. This gives more weight to points within the core than schemes such as those proposed by Pegrum (2006) and Shah (2005) which are based on the radial distribution of circulation. As a consequence, the Bertényi scheme is less prone to interference from neighbouring vortices. The following form of the equation for the tangential velocity for an $\mathrm{L}-\mathrm{O}$ vortex can readily be derived from the one quoted in the "Appendix".

$\mathbf{u}_{\theta}(r, \theta)-\mathbf{u}_{P_{V}}\left(x_{0}\right)=\frac{\Gamma_{0}}{2 \pi r}\left(1-\mathrm{e}^{\frac{-r^{2}}{4 t}}\right)$

This equation is employed in this study for the correlations with the measured SS-PIV data using the MATLAB lsqcurvefit multi-variable least-squares fitting function which was found to converge very rapidly. All of the defining parameters, $\Gamma_{0}, r_{\text {core }}$ and $\mathbf{x}_{0}$, can be evaluated simultaneously using this function for each vortex. This yields updated values for the centre coordinates, revising the initial location which was inferred from the centroid of the $\gamma$ distribution. The results are sensitive to the size of the interrogation window relative to the extent of the vortex and it was evident that in formulating an automated data processing routine it was important to maintain a region that was large enough to ensure accurate results. The problem is exacerbated when a flow has multiple vortices that are close together. It is then more difficult to determine their properties accurately because of the distorting influence of the neighbours. One way to overcome this is to shrink the area from which data are taken when seeking the correlation with the idealised model. Thus, it is useful to have a measure of how much this can be done without compromising the accuracy.

To give an indication of the sensitivity in deriving properties of a vortex to a reduction of the size of the interrogation region, a numerical study has been done using real data from a vortex measured in the experiment to be described later in this paper. It is from the persistent vortex for which results of its trajectory will be shown in Fig. 12, and designated as vortex A in Fig. 13. Estimates for the various vortex parameters derived using different sized evaluation region are shown in Fig. 2. A square region of $n \times n$ cells has been used centred on the vortex axis. The core diameter is indicated on the plots to relate the mesh extent to the vortex size.

From these plots it is evident that the properties are recovered to a good approximation once the interrogation window exceeds the size of the vortex core by about $30-40 \%$. The derived core radius is shown in Fig. $2 \mathrm{~b}$ in terms of $4 v t$. which for an $\mathrm{L}-\mathrm{O}$ vortex is equal to $0.8902 \sqrt{r_{\text {core }}}$. Bertényi (2001) define a function $R^{2}$ that indicates how well the predicted profile compares with the experimental data, $R^{2}=1$ implying a perfect match. This property was monitored throughout the automatic data processing as a quality check and is shown in Fig. 2c. Plot (d) shows a comparison of the vortex centre coordinates $y$ and $z$ derived in two ways-from the centroid of the $\gamma$ distribution (which overcomes the difficulty in determining the peak of the flat-topped curve) and from the idealised vortex fit. It can be seen that for both coordinates essentially the same values are obtained for this vortex using either method once the extent of the interrogation region is adequate.

\section{Extension of the vortex detection method}

Berdahl and Thompson (1993) and Jeong and Hussain (1995) showed that improvements can be made in detecting vortices by splitting the velocity gradient tensor into its symmetric $\mathbf{S}$ and antisymmetric $\boldsymbol{\Omega}$ parts, these representing the strain and rotation elements respectively. By subtracting the effect of strain from the measured vectors, regions 


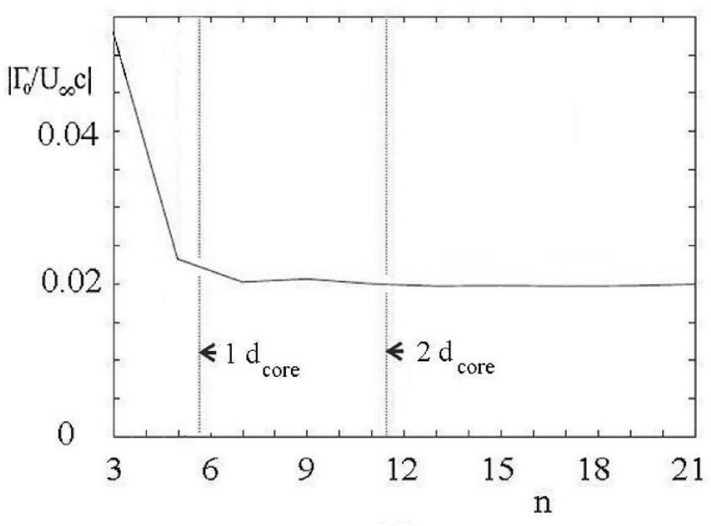

(a)

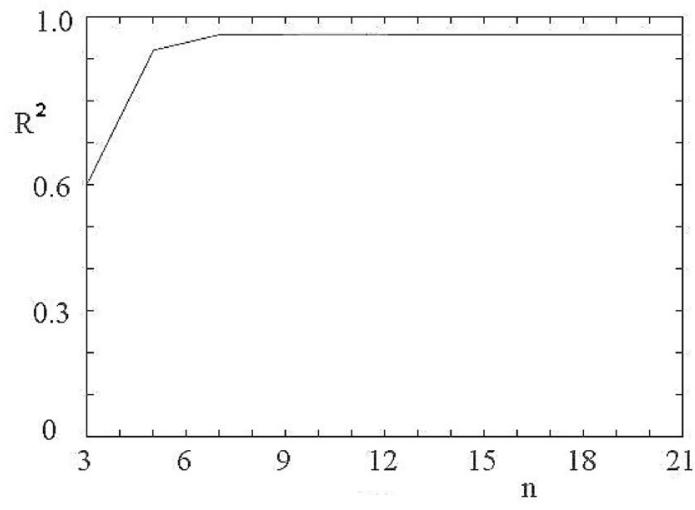

(c)

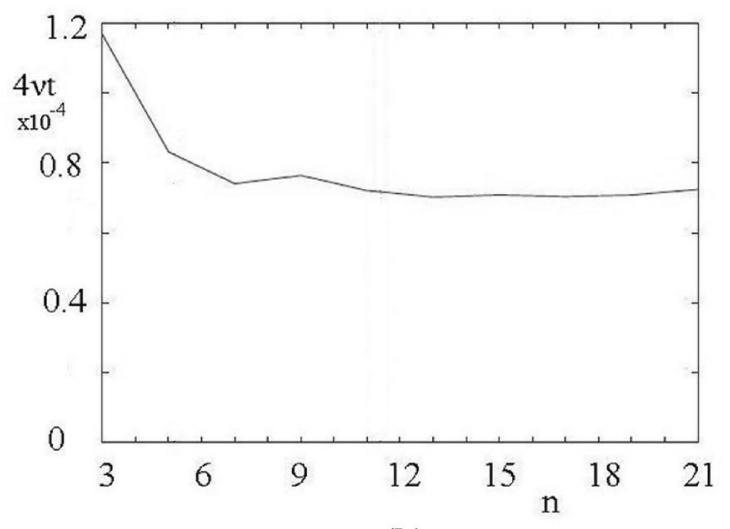

(b)

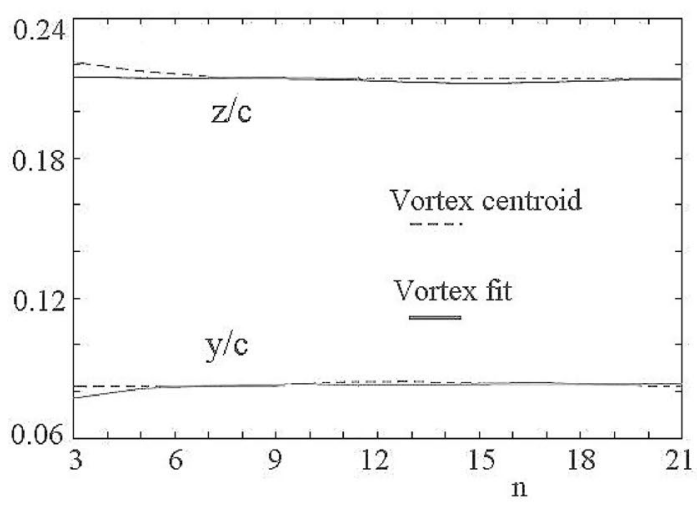

(d)

Fig. 2 Plot showing a the circulation, $\mathbf{b}$ core radius, $\mathbf{c} R^{2}$ and $\mathbf{d}$ centre coordinates derived for a measured vortex using variable size of interrogation region. (The core radius is plotted as $4 v t$ which for an $\mathrm{L}-\mathrm{O}$ vortex equals $0.8902 \sqrt{r_{\text {core }}}$ )

of swirling flow can thus more readily be identified. Graftieaux et al. in their formulation subtracted a constant $\mathbf{u}_{P}$ from the velocity to minimise the strain, making the detection scheme Galilean invariant. However, by treating $\mathbf{u}_{P}$ as uniform over $S$ the possibility of any velocity gradients in the background shear within the region were ignored. For flows where vortices are in close proximity, this is not a good assumption since each neighbouring vortex induces a velocity that varies with distance. It follows that improvements can be made by explicitly acknowledging the variable contribution to $t$ shear that is attributable to the neighbouring vortices. To a good approximation this can be done by replacing each of these vortices with an equivalent point vortex located at the same position. The overall contribution to the shear velocity at any point $M$ from $N$ neighbouring vortices is obtained by summing the induced velocities, that is $\mathbf{u}_{P_{\mathrm{V}}}=\sum_{N} \mathbf{u}_{\theta, i} \sum_{N} \Gamma_{i} / 2 \pi r_{i}$, where $\Gamma_{i}$ is the circulation of each neighbouring vortex $i$ and $r_{i}$ is the distance between its centre and the point $M$. Thus, after first processing the data set using the Graftieaux scheme and obtaining an optimised match with an idealised $\mathrm{L}-\mathrm{O}$ vortex, it is possible to reevaluate it, replacing the constant value of the convective velocity $\mathbf{u}_{P}$ with a varying velocity distribution that is derived by combining the induced velocity of the other vortices. This yields updated values for the strength, core radius and position of each vortex. This procedure can be repeated iteratively to redefine each vortex and in practice it rapidly converges. If there is a shear in addition to that induced by the vortices it can be also be subtracted.

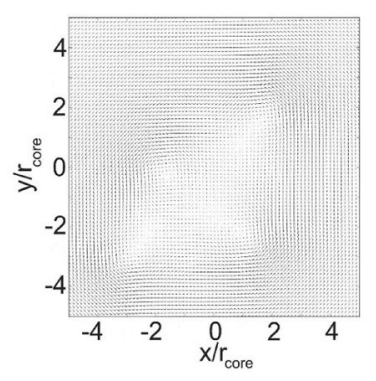

(a)

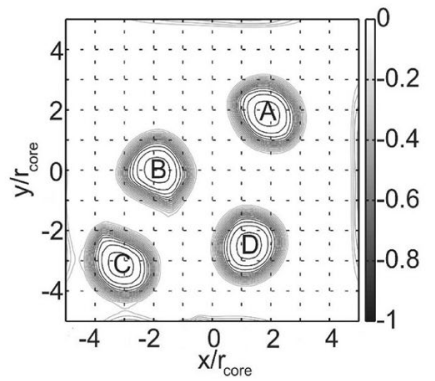

(b)
Fig. 3 a Vectors generated for the four vortices. b The corresponding contours of $\gamma$ 


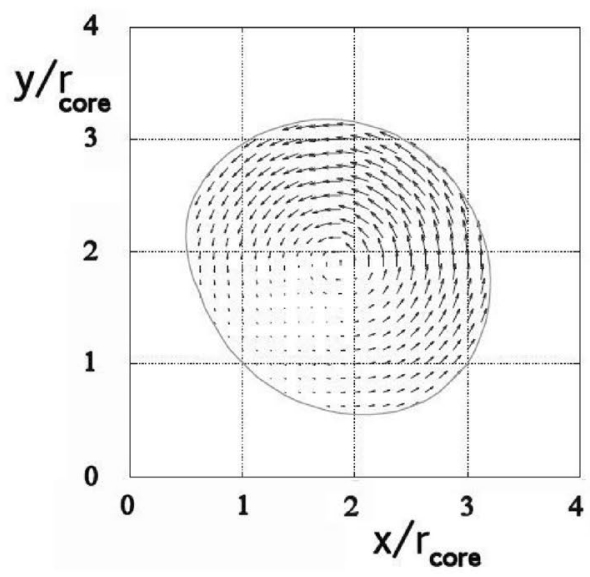

(a) no correction

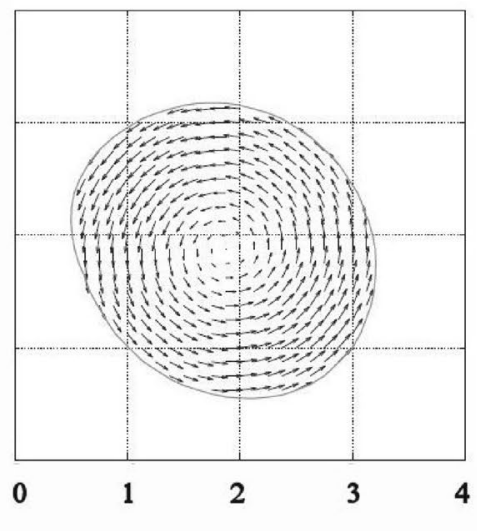

(b) uniform shear

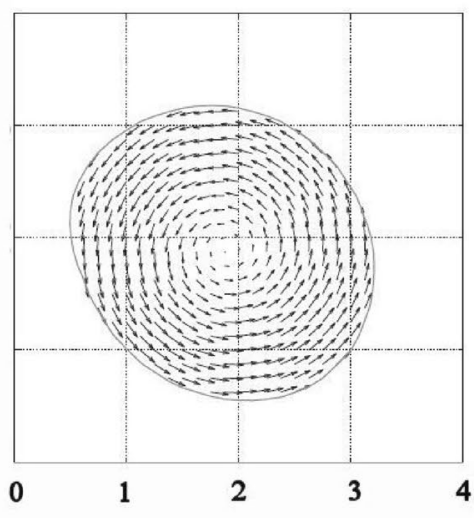

(c) vortex induced velocity

Fig. 4 The effect of the velocity correction on the vectors for vortex A within the $\gamma=|0.3|$ boundary

The effectiveness of this procedure is demonstrated in the following example. An array of artificial velocity vectors (see Fig. 3a) has been generated for a cluster of four randomly placed $\mathrm{L}-\mathrm{O}$ vortices. This has been done without adding noise to enable a more precise evaluation of the accuracy of the method. Figure $3 \mathrm{~b}$ shows the corresponding $\gamma$ contours obtained using the Graftieaux function from which the location of each vortex can recognised.

To evaluate the circulation, it is necessary to set a limit to the region from which data are taken. This can be done by defining an arbitrary circular limit but in this instance, it has been achieved by setting a boundary defined by $\gamma \geqslant|0.3|$ which takes some account of the distortion of the vortex due to its neighbours. (This would have corresponded to $1.3 r_{\text {core }}$ for an L-O vortex). The effect of the corrections on the velocity data is shown in Fig. 4. Image (a) illustrates the uncorrected velocity data for this region. The effect of subtracting the average shear, shown in (b), yields a pattern of vectors that more closely resemble a recognisable vortex. Incorporating the non-uniform vortex induced shear velocities is plotted in (c) and although it is difficult to distinguish this from that in (b), it yields a much-improved estimate of the circulation recovered from the $\mathrm{L}-\mathrm{O}$ vortex correlation. Table 1 shows the percentage errors in the circulation obtained from the correlation with various corrections for the shear velocity. After iteration using the induced velocities in which the values of the circulation and position of each vortex are readjusted once, it can be seen that the errors diminish rapidly. With ten iterative steps the circulation for all of the vortices is recovered to within better than $0.1 \%$ of the correct value. This is achieved despite having used the approximation of point vortices to replace the regions of distributed vorticity of the original $\mathrm{L}-\mathrm{O}$ vortex.

It is accepted that there are limitations in the use of this procedure and if the vortices are very close and destructively interacting or near to merging this is not an appropriate approach. Seeking a correlation with an idealised vortex or calculating the induced velocity from neighbouring vortices assuming point vortices will not yield the correct results. Judgement as to whether to use these procedure has to be made in the light of knowing the structure of a flow possibly from raw PIV data. However, for vortices spaced at distances comparable to those shown in Fig. $3 b$ the methods proposed have been shown to work well.

\section{Effect of inclination of the vortex axis}

When studying flows involving multiple vortices, such as the one about to be described, more often than not the PIV laser sheet is not perpendicular to the vortex axis. For a cluster of spiralling vortices, it is not possible to have more than one core perpendicular to the sheet. Thus, it is instructive to assess any error in determining the properties of the vortices when the laser sheet is tilted with respect to the vortex axis, say by an angle $\phi_{1}$.

For an axisymmetric vortex with no axial velocity, tilting the laser sheet produces two effects that influence its inferred properties. Circular paths about its axis are stretched and appear as ellipses with the major axis increasing by a factor $(\sec \phi)$. Although the length of the minor axis is unchanged,

Table 1 The percentage error in evaluating the circulation of each vortex

\begin{tabular}{llrrr}
\hline Correction & $A$ & \multicolumn{1}{l}{$B$} & \multicolumn{1}{l}{$C$} & \multicolumn{1}{l}{$D$} \\
\hline None & 5.54 & -2.20 & -11.20 & -15.30 \\
Average velocity & 0.07 & -2.20 & -11.08 & -18.18 \\
Induced velocity & 0.59 & 0.23 & 0.72 & 0.47 \\
\hline
\end{tabular}




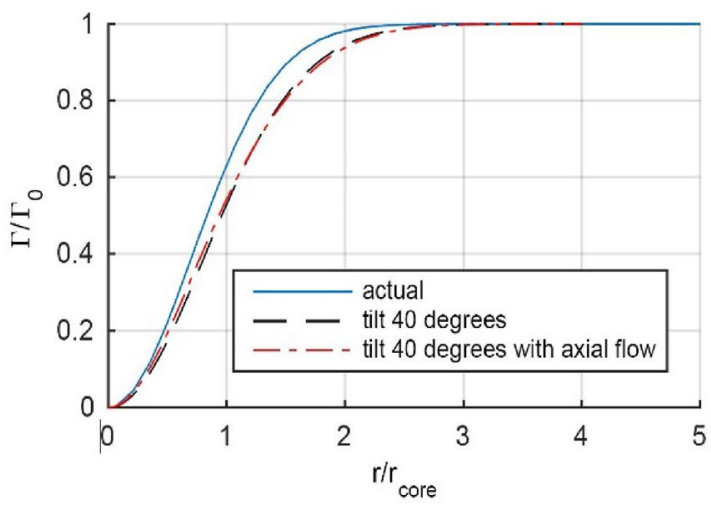

(a)

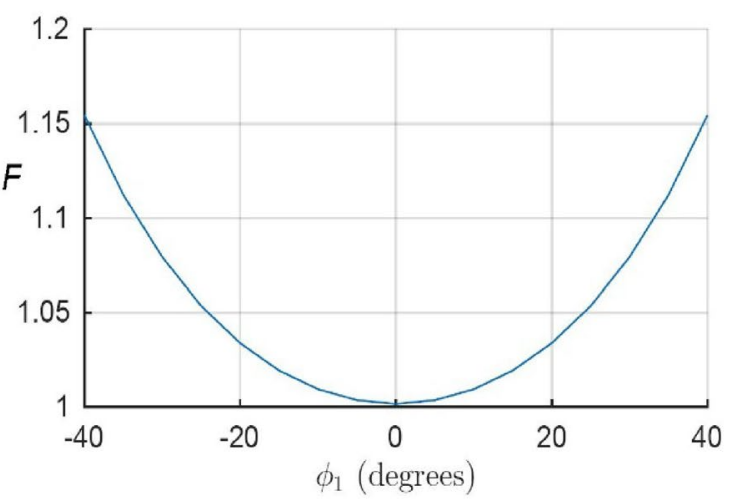

(b)

Fig. 5 a Circulation distributions obtained for a synthesised L-O vortex with the laser sheet tilted $40^{\circ}$; $\mathbf{b}$ the error in the inferred radius due to tilt for a vortex with no axial velocity

the tangential velocities along it are distorted and they appear to be reduced by a factor of $(\cos \phi)$. This change diminishes to zero as one moves around the vortex towards the major axis. If the circulation is evaluated by integrating along some circular contour surrounding the vortex on the laser plane, the value obtained will be in error because of these effects. However, in this study where the properties have been inferred from a correlation with an idealised L-O vortex, the outcome is not the same.

To quantify the effects of tilting, a numerical simulation has been performed using an array of synthesised velocity vectors that represent an isolated $\mathrm{L}-\mathrm{O}$ vortex initially with no axial velocity. These were spaced at $0.1 r_{\text {core }}$ intervals on a square mesh. To simulate the titled laser sheet the array was transformed to how it would appear on an inclined plane representing the laser sheet and then analysed using the $\gamma$ distribution and by finding the best correlation with an $\mathrm{L}-\mathrm{O}$ vortex using the Matlab least squares function. This simulation has been repeated for a range of tilt angles up to $40^{\circ}$. Figure $5 \mathrm{a}$ shows the results obtained for the radial distribution of the circulation compared with the original vortex when the plane is tilted by $40^{\circ}$. Although the curve is stretched in the radial direction it asymptotes to the correct value of the circulation and the true position of the centre is found. However, as shown in Fig. 5b, the apparent radius is over estimated and the vortex is enlarged by the factor $F=r_{\text {estimated }} / r_{\text {core }}$.

This analysis has been performed without any additional axial velocity component. But for experiments in wind tunnels there is a large free stream component in this direction and thus the question arises as to whether this influences the accuracy in determining the vortex properties if the laser plane is tilted. Thus a similar analysis has been performed with a constant added axial component of velocity parallel to the vortex axis arbitrarily set equal to $V_{\vartheta, \max }$ to illustrate the effect. The result derived for the circulation has been added to Fig. 5a and it is clear that including a constant axial velocity results in no measurable difference due to tilting other than that seen in the example without axial flow. The circulation is recovered exactly as before providing the region is extended to about $3 r_{\text {core }}$, the vortex centre location is unaffected by the tilting and the value of the core radius is increased by exactly the same amount as was seen without the axial velocity component. The constant axial component does not add any vorticity to the flow and which explains why the inferred value of the circulation about the vortex remains unaltered.

However, real vortices shed from lifting surfaces do not have a constant axial velocity, but generally exhibit a wake like core when viewed in a frame fixed with the body producing the vortex. To illustrate the effect of tilting the laser plane when measuring one such vortex, the same analysis has been repeated on an array of simulated vectors corresponding to an $\mathrm{L}-\mathrm{O}$ vortex but with an added Gaussian distribution of axial velocity centred on the axis given by $V_{x}=A \mathrm{e}^{-2\left(r / r_{\text {core }}\right)^{2}}$ to approximate what is observed. Initially the simulation was performed with zero axial velocity away from the vortex which corresponds to what is observed when a vortex is generated in a towing tank experiments such as the one about to be described in this paper where the fluid is initially at rest. The L-O vortex has a maximum swirl velocity $V_{\vartheta, \max }$ at the core radius. Arbitrarily setting $A=V_{\vartheta \text {,max }}$ produces a distribution of axial velocity that has a peak on the vortex axis equal to the maximum swirl velocity and which drops to $13.5 \%$ of this value at the edge of the core. As in the previous examples fitting the idealised vortex recovers the circulation exactly. Similar errors arises in the inferred core radius, $F$ being 1.138 at $\phi_{1}=40^{\circ}$ compared with 1.154 seen previously. However, what is different is that there 
Fig. 6 The error in locating the centre of vortex with a Gaussian axial velocity distribution for various angles of tilt
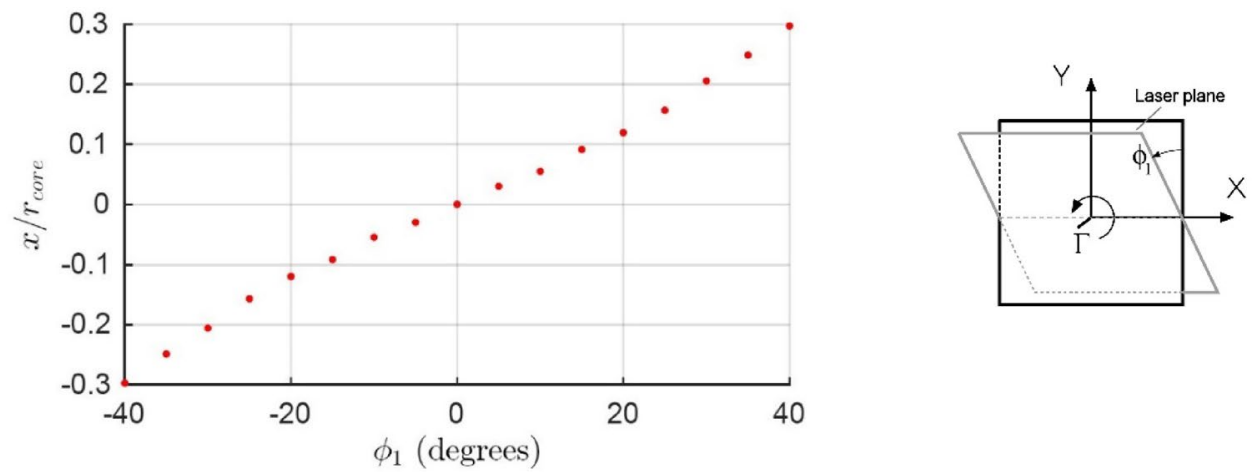

is a sizeable error in locating the centre of the vortex as shown in Fig. 6.

A second simulation has been performed representing measuring a vortex with an axial velocity deficit in a wind tunnel where there would be an external uniform axial flow. The core deficit is given by the expression $V_{x}=-V_{\theta, \max } \mathrm{e}^{-2\left(r / r_{\text {core }}\right)^{2}}$ which is combined with a uniform axial velocity representing the free stream arbitrarily set at $V_{\vartheta \text {,max }}$. It is found that the same errors occur in assessing the vortex characteristics for this flow as in the previous example just considered. It is thus concluded that adding an axial velocity field that is irrotational - that is free from any vorticity — does not change the errors in the deriving the vortex properties from a PIV survey when the measuring pane is tilted. However, if there is vorticity associated with the axial flow distribution, such as with the Gaussian distribution, significant distortions will occur in the $\gamma$ distribution and sizable errors arise in determining the vortex centre location.

The examples presented here are not intended to quantify these errors for all situations but instead, to provide guiding principles and it is evident that errors can arise, in particular, in determining the location of a vortex, if the laser plane is tilted. The conclusions are relevant to both two- or three-dimensional PIV data and presuppose that the inclination of the vortex to the measuring plane, something that can be determined by tracing the vortex trajectory between planes spaced along the vortex axis, is known. With three-dimensional PIV data it is possible to resolve and interpolate the velocity vectors into an inclined plane that is truly perpendicular to the vortex axis before evaluating its properties. While the above analysis suggests that if the laser plane tilt is not excessive the circulation and core radius can be obtained with good accuracy without doing this, it does suggest that this procedure is necessary if the precise location of the vortex centre is being sought.

\section{Experimental examples}

To illustrate these adaptations to the data processing when using SS-PIV, the results are analysed from tests that have been conducted on a representative $50 \%$ scale model of the front wing and wheel of a Formula 1 car (Morgan 2010). The wing has a complex geometry featuring multiple end plates and inboard fences, which produce a cluster of vortices and shear layers. It is situated just upstream of the front wheel (see Fig. 7 insert) which has a large and very unsteady wakes that randomly perturb the paths of these vortices as they pass around it. The vortices play a very important role in determining the aerodynamic performance of the car and hence it is important for its designer to be able to determine their trajectories and properties as they propagate over the rest of the car. Because of the unsteadiness is only possible to investigate their behaviour using instantaneous measurements, that is, it necessitates the use of SS-PIV.

The tests were conducted in a towing tank shown in Fig. 7. This is $1.0 \mathrm{~m}$ wide and the water has a nominal depth of $0.9 \mathrm{~m}$. One-half of a front wing was used which has a semi-span of $350 \mathrm{~mm}$ and a chord width c including its flap of $225 \mathrm{~mm}$. This was suspended vertically below a servo-driven carriage and after an initial acceleration it was towed steady through the water at $3 \mathrm{~m} / \mathrm{s}$. The Reynolds number based on $c$ was $6.4 \times 10^{5}$ which is close to that obtained by $\mathrm{F} 1$ teams in routine wind tunnel tests using $60 \%$ scale model cars. The streamwise coordinate $x$ is measured from the leading edge of the wing.

The wing was positioned near a side wall of the tank at a distance corresponding to the height it would be above the ground when fitted to the car. A large horizontal flat 


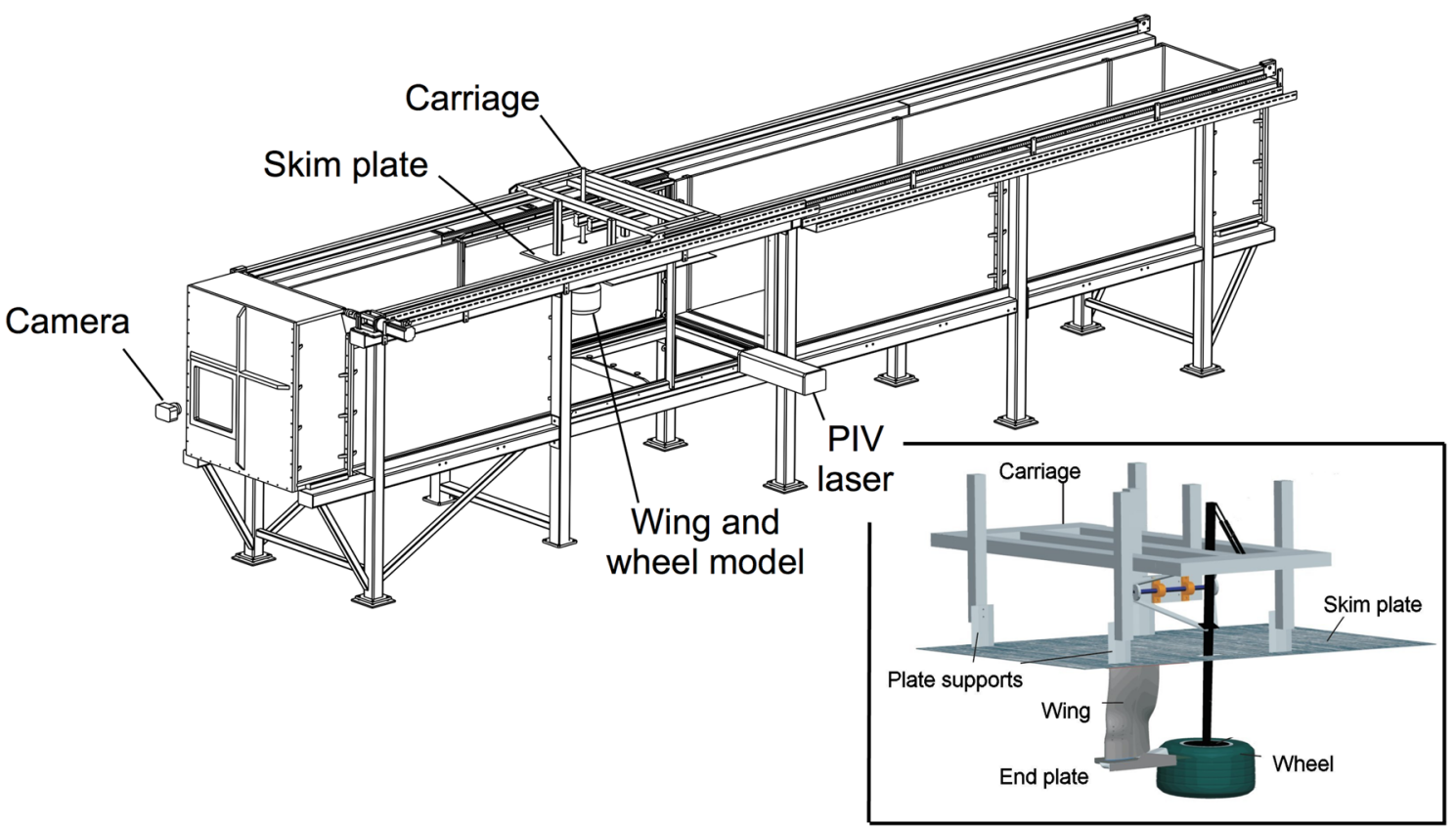

Fig. 7 The towing tank and details of the model

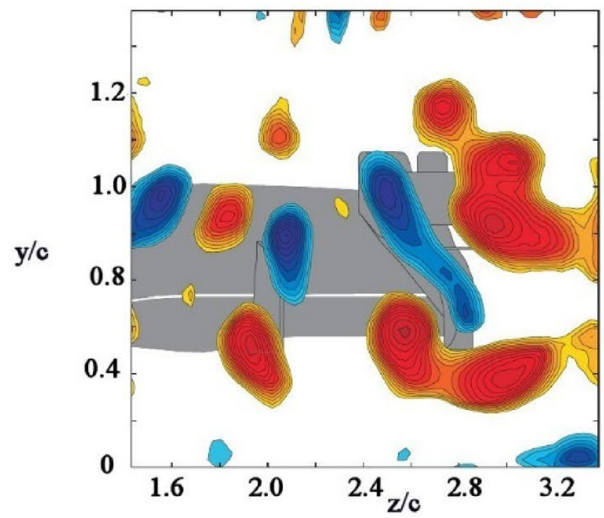

(a)

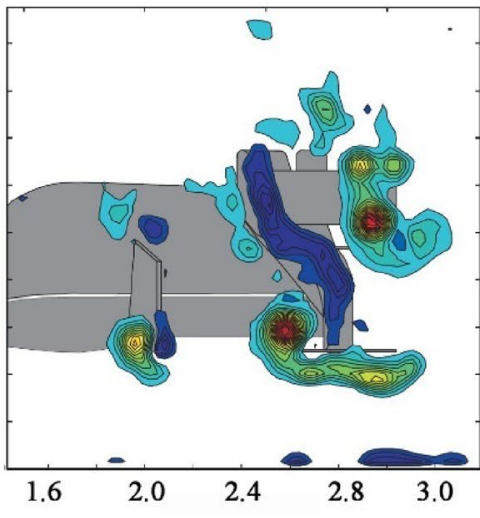

(b)

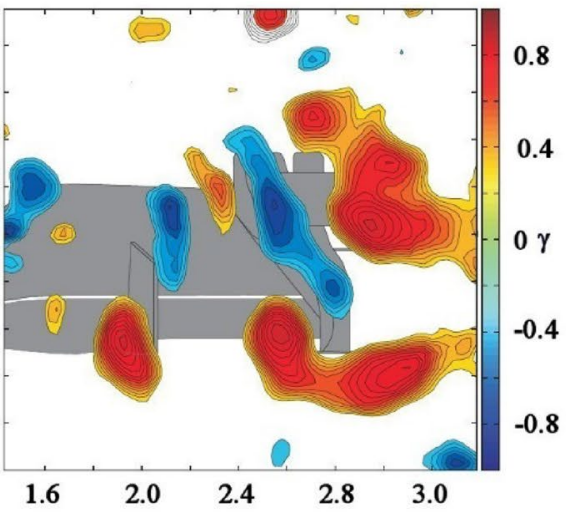

(c)

Fig. 8 A comparison between the $\gamma$ contours in the wake of the wing obtained at the end plate trailing edge; a from the averaged multiple-shot PIV surveys; $\mathbf{b}$ the corresponding vorticity; $\mathbf{c}$ the $\gamma$ contours from a single-shot survey

skim plate was mounted beneath the carriage spanning the tank just below the free surface. This acted as an image plane for the half span wing as well as preventing the formation of excessive surface waves in the vicinity of the model.

An LaVision FlowMaster4S DPIV system was used which consists of a Pegasus Nd:YLF double cavity laser and a Fast-Cam Ultima APX camera. This has a resolution of $1024 \times 1024$ pixels when operated at a frame rate of $500 \mathrm{~Hz}$. With the model being towed at $3 \mathrm{~m} / \mathrm{s}$, this produced results for successive $y-z$ planes perpendicular to the model's motion spaced at $6 \mathrm{~mm}$ intervals. The field of view was approximately $220 \mathrm{~mm}$ square giving a spatial resolution of 4.65 pixels $/ \mathrm{mm}$. The camera was positioned at the end of the tank and was about $3.5 \mathrm{~m}$ from the laser sheet. Thus, the angle between the camera optical axis and points within the field of view was below $2.5^{\circ}$ and any remaining parallax error is compensated for within the PIV calibration software. Seeding was done using neutrally buoyant particles (Vestsint 7182) with an average diameter of 30 microns. Due to diffraction in the camera lens and the effect of photographing through over $3 \mathrm{~m}$ of water, the particle images were observed to spread over between 3 and 4 pixels and no peak locking was detected. Systematic errors in the velocity 
were determined following the procedures outlined in Morgan (2010) and were estimated to be no more than $1.7 \%$. They are considerably smaller than the velocities induced by the nearby vortices (see Table 1). This estimate for the errors does not, however, take into account the influence of local velocity gradients within the interrogation window nor out of plane movement of particles. However, with towing tank experiments where there is no motion between the ambient fluid and the laser sheet, the latter error is significantly mitigated.

\subsection{Wing alone tests}

Initially tests were performed on the wing on its own. This has a steady flow and hence the SS-PIV results can be compared with those from averaging several runs since it is reasonable to assume the later provides a true representation of the flow. Figure 8a shows a comparison of the $\gamma$ contours for the near wake of the wing at the end plate's trailing edge from the averaged results with those taken from the single shot survey (c). Plot (b) shows the vorticity derived from the averaged data which possibly gives a clearer idea of the complex vortex structure. The corresponding plot of vorticity from the SS-PIV was too noisy to be useful. For this example, an $11 \times 11$ point interrogation window was used to calculate $\gamma$ which ensured that all the points within an adequate area for each significant vortex were included in the analysis. Although there are some anomalies between the two $\gamma$ plots, the significant vortices are detected in the single-shot survey. The $\gamma$ method simply locates rotating structures and is unable to discriminate between strong and weak vortices. Any spurious peaks will be rejected when it comes to matching to an $\mathrm{L}-\mathrm{O}$ vortex. It is concluded that it is feasible to establish the location of the important vortices in a flow from a carefully performed SS-PIV test. From here it is possible to obtain the values of the circulation $\Gamma_{0}$, the core radius $r_{\text {core }}$ and the precise centre location $\mathbf{x}_{0}=\left(y_{0}, z_{0}\right)$ for each of the significant vortices from a correlation between the measurements and a matched $\mathrm{L}-\mathrm{O}$ vortex.

In the present experiments, the PIV surveys were performed at a high repetition rate. Thus, as the model passed the laser sheet the survey planes were closely spaced relative to the model, separated by only $6 \mathrm{~mm}-$ a distance equal to only $2.7 \%$ of $c$ and about $1 / 10$ of the vortex core diameter. For most of the wake the position and properties of each vortex changed very little between neighbouring planes. Exceptions to this would be if vortex breakdown occurred or if the vortices merged. The close proximity of the survey planes was exploited in two ways. Firstly, when implementing the iterative vortex matching scheme, it was possible to make a very good initial prediction of where the centre would be in the next plane by assuming that it has moved from plane $N$

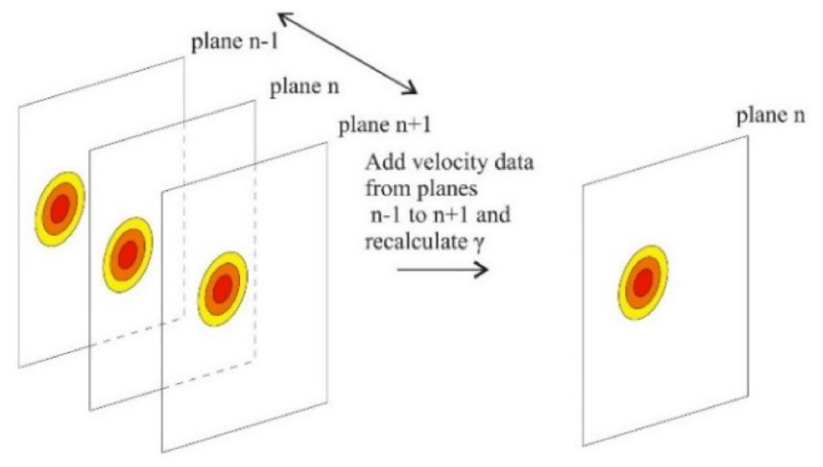

Fig. 9 Averaging of the PIV data

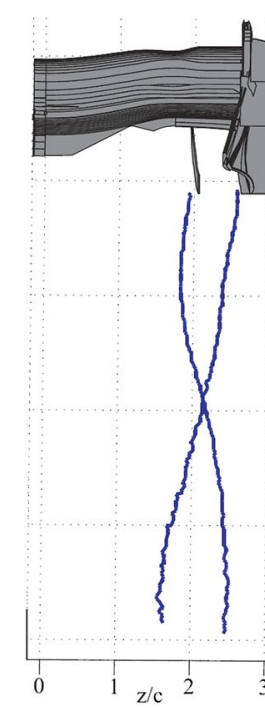

(a)

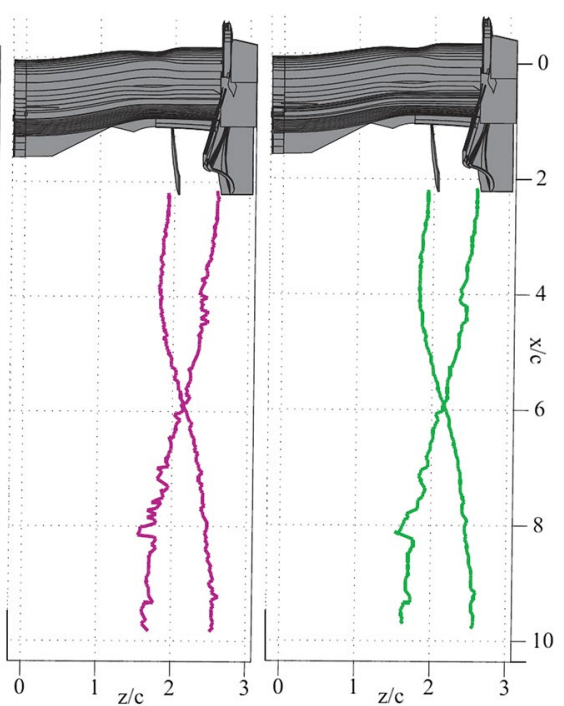

(b)

(c)
Fig. 10 The plan view of examples of the trajectories of the two most dominant vortices shed from the wing. a Trajectories averaged from multiple surveys; $\mathbf{b}$ single plane SS-PIV; $\mathbf{c}$ from 3-plane averaged SSPIV

to plane $N+1$ due solely to the mean transverse velocity at the first plane. That is, its position is assumed to be

$\mathbf{x}_{o, N+1}(y, z)=\mathbf{x}_{o, N}(y, z)+\delta \times \mathbf{u}_{P_{\mathrm{V}}}\left(\mathbf{x}_{0}\right)_{N}$,

where $\delta$ is the time separation between the two planes. Using this value accelerates the convergence, but more importantly it also ensures that the automatic data processing followed the same vortex from plane to plane. The values of $\Gamma_{0}$ and $r_{\text {core }}$ from the previous plane were also used as initial values for the iteration.

Secondly, since the planes are closely spaced it is reasonable to assume that each vortex will change only very little from one to the next. Thus, it is legitimate to average the SSPIV data over a small cluster of adjoining planes to improve the signal-to-noise ratio. This is effective particularly in 
Fig. 11 A sample comparison between the instantaneous and 3-plane averaged results for the circulation $\left(\Gamma_{o}\right)$ and the core radius expressed as $4 v t$ where $r_{\text {core }}=1.121 \sqrt{4 v t}$.
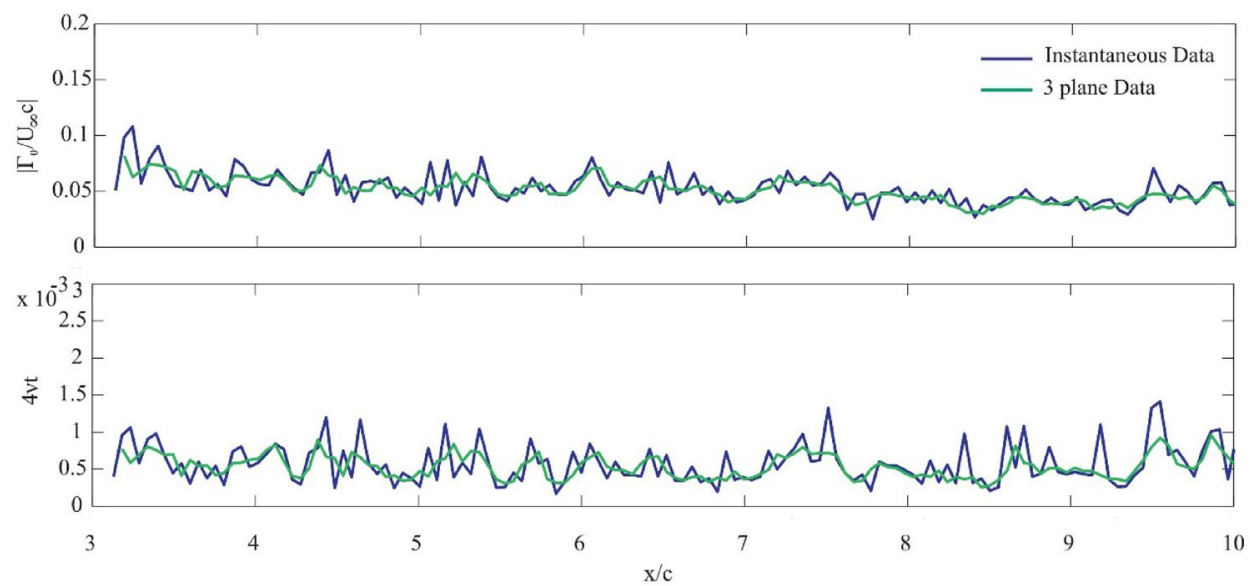

evaluating the circulation. 3-plane averaging was adopted in these tests as depicted in Fig. 9 but more planes could readily have been used, with the possible option of employing variable weighting. The number of planes judged as appropriate has to be decided from a knowledge of the likely changes in the vortex over the distance they span. This can be ascertained from an initial examination of the single plane results, using a criterion set by specifying acceptable variations. However, it is also necessary to acknowledging that data from SS-PIV surveys will fluctuate from plane to plane which frustrates automating this decision and inevitably the choice will also have to rest to some extent on a knowledge of the flow physics. Before averaging, the origins of the downstream and upstream data sets were shifted by $\boldsymbol{x}_{0, N+1}-\boldsymbol{x}_{0, N}$ or $\boldsymbol{x}_{0, N}-\boldsymbol{x}_{0, N-1}$, respectively.

The paths obtained for the two most dominant vortices shed from the wing alone are shown in Fig. 10. Image (a) shows the trajectories that are derived from averaging the multi-shot PIV data which can be viewed as a true representation of the flow. The other two examples show the results from SS-PIV surveys. In (b) the trajectories obtained by processing individual plane are shown and in (c) the slightly smoother results for the 3-plane averaging are plotted.

The real advantage of employing the 3-plane averaging is more evident in the results for the circulation and the core radius. Figure 11 shows typical plots of these properties of the fence vortex shown Fig. 10 and while fluctuations still occur after the averaging, the curves are noticeably smoother. The standard deviation for the circulation relative to the RMS value has been reduced by a factor of 1.9 and, although not shown here, the occasional false values are eliminated. Adding more planes would of course give added smoothing but may risk hiding some genuine variations.

\subsection{Wing and wheel tests}

A second set of tests was conducted adding a 50\% scale wheel which was correctly positioned relative to the wing.
The wheel's inflatable tyre was pressed against the wall so that it rotated and thus by moving the wing-wheel assembly along the wall it was possible to achieve a true simulation of the effect of a stationary ground on the flow.

Being a bluff body, the wheel produces an unsteady wake. The flow passing over its top and sides gives rise to a pair of large, unstructured longitudinal vortices which are swept downwards within the wake towards the ground further downstream. These we refer to as the 'top wheel vortices'. A secondary pair of far steadier vortices appear close to the ground. They emerge from the region just ahead of where the wheel comes in contact with the ground and pass either side around the bottom of the wheel. This pair, referred to here as the gap vortices, has the appearance of a conventional horse-shoe vortex that would form around the base of an object standing within a shear layer but its origins are very different. Relative
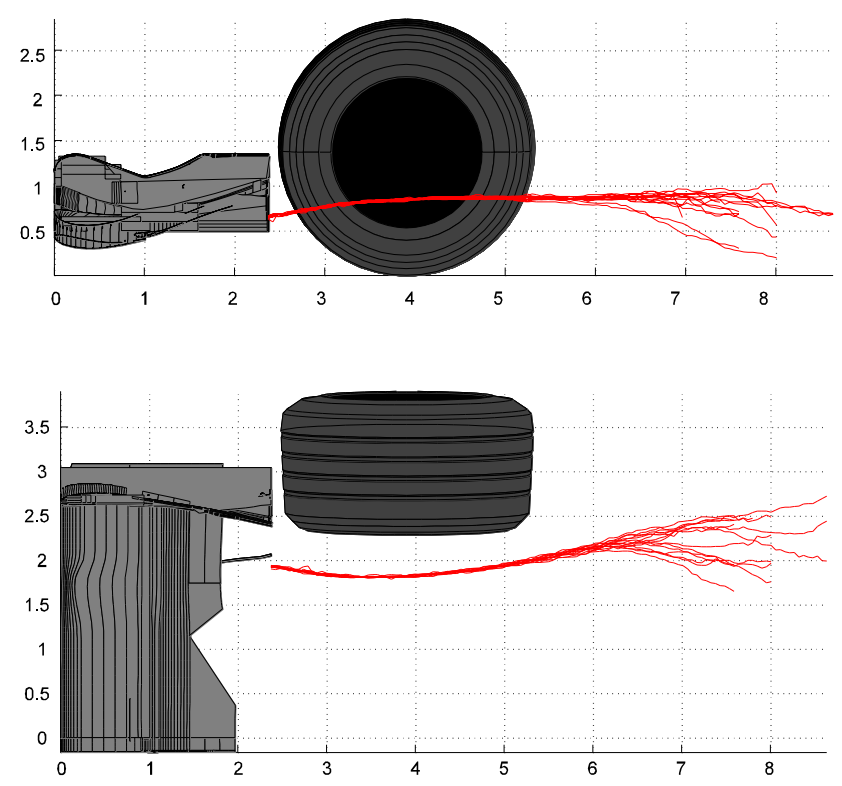

Fig. 12 The trajectories for the mid-span fence vortex for 14 different tests 
to the wheel, both the ground and the air approach it at the same speed. Thus, without any relative motion, no boundary layer forms on the ground until very close to the wheel and insufficient shear is generated to produce a significant horseshoe vortex. The origin of these secondary vortices is instead attributable to the convection towards the ground of vorticity generated within the boundary layer formed on downwardmoving front surface of the tyre. The viscous action of squeezing the air just ahead of the contact patch as the tyre rolls forward also contributes to their formation.

In the examples shown so far in this paper, the vortex trajectories have been essentially steady. However, adding the wheel causes their paths to be randomly perturbed once they come within the influence of its unsteady wake.

Examples of the trajectories obtained from the SS-PIV surveys using 3-plane averaging are shown in Fig. 12, in this case, for the vortex that is shed from the wing's mid-span fence. In this plot 14 samples taken at different times have been superimposed to illustrate the variation in the paths taken. As the vortex passes the side of the wheel, initially it follows more or less the same trajectory as the gap vortex which predominantly effects it is relatively steady. However, once it comes within the influence of the wheel's unsteady wake, the path beginning to deviate and at a distance of about one wheel radius downstream of the wheel this divergence becomes more pronounced. It can be seen that the trajectories depart significantly from what would be the mean path. With repeated tests, the probability distribution of the location of each vortex can be ascertained. Similar results were obtained for the other vortices shed from the wing.

Figure 13 gives a sample representation showing the core radii of the major vortices detected in the flow for a typical wing-wheel arrangement, the different colours indicating the positive or negative sense of rotation. The mid-span fence produces the vortex labelled (A) which exhibits a near constant core radius as it propagates downstream until eventually it becomes enmeshed in the wake downstream of the wheel. Samples of the circulation and core radius of vortex A have already been shown in Fig. 11. Another strong vortex (C), of the same sign, is shed from the bottom tip of the wing's end plate. This propagates nearer the ground and as a consequence interacts with the inboard gap vortex (B) which also is of the same sense. These subsequently begin to merge, but then break up as they come under the influence of the unsteady wheel wake. The presence of the very unsteady top wheel vortices shed into the wake can also be detected although they appear to lack any clear structure.

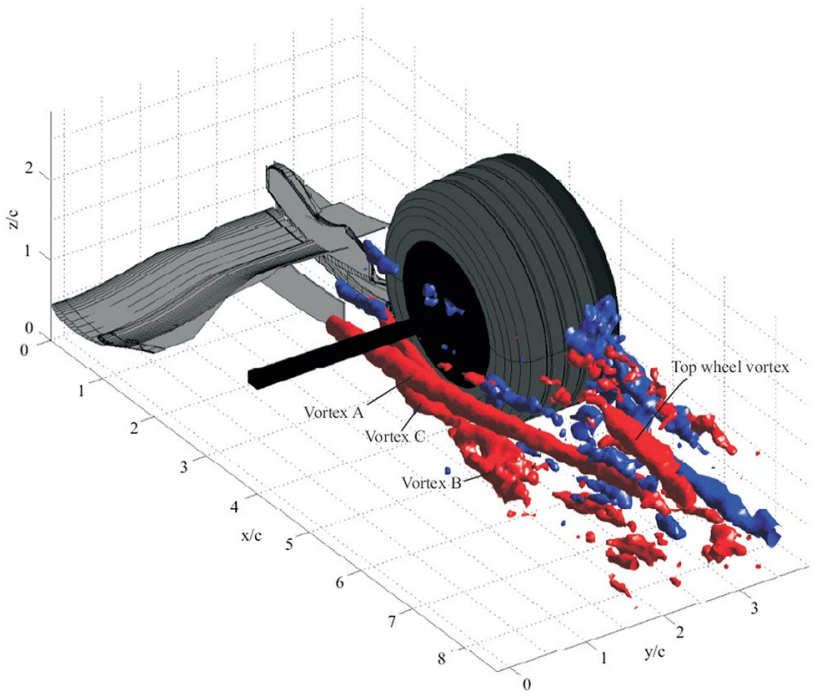

Fig. 13 A plot showing the core radius of all of the vortices present in the wing-wheel flow

\section{Conclusions}

This study demonstrates that single-shot PIV test can be used to study vortices in situations where their paths fluctuate due to unsteady influences elsewhere in a flowfield. The techniques described have been applied to PIV velocity measurements obtained from tests performed in a towing tank to investigate the vortex wake of a F1 car's front wing and wheel. The wheel, situated just downstream of the front wing, has a large unsteady wake that significantly perturbs the paths of the trailing vortices shed from the wing.

It is concluded that from carefully executed experiments, good results can be obtained for the trajectories and properties of a cluster of trailing vortices from single-shot surveys using an automated data processing procedure. The vortices were initially located using Graftieaux scheme before determining their strengths and core radius by seeking the best correlation with an idealised L-O vortex model. However, the constant shear velocity assumption on which that scheme is based was replaced by an improved estimate taking into account the spatially varying induced velocity due to neighbouring vortices. An iterative scheme that can be applied to any PIV data was implemented to redefine the position and strength of each of the vortices within the cluster from the initial estimates. Using a synthesised set of velocity vectors for a cluster of four vortices it has been demonstrated that the vortex strengths can be recovered to an accuracy of better than $0.1 \%$ after ten iterations using this procedure. For this specific example, which may or may not be truly typical, this is an error of more than an order of magnitude less than that obtained using the Graftieaux scheme. 
Operating the PIV system at a high frequency and using a towed model yielded velocity data in cross-flow planes that were closely spaced in the streamwise direction relative to the model. Since each vortex changed only slightly between successive planes, it was considered legitimate to average the PIV data over adjacent planes to improve the signal-tonoise ratio. This technique has potentially wider application, for example, when identifying vortex structures in turbulent flows from surveys closely separated in time. For the present tests 3-plane averaging was deemed sufficient but more planes could be contemplated. An added advantage of having closely spaced data planes is that it allows the results from one plane to be used as a very good starting point when seeking the correlation with an $\mathrm{L}-\mathrm{O}$ vortex for the next plane. This accelerates the convergence and ensures that the same vortex is followed in the automatic data process.

Estimates are included in the paper for the error incurred in evaluating the properties of a vortex when the PIV laser plane is not perpendicular to its axis. Assessments have been made of the errors in evaluating the circulation, core radius and centre location using the $\gamma$ method and seeking the closest correlation with an idealised model vortex for several situations. Firstly for a synthesised L-O vortex with and without a superimposed constant axial velocity was studied and then for a vortex with a variable axial velocity deficit representing a real vortex, again with and without a superimposed constant axial velocity. In all situations the circulation was recovered accurately but the inferred radius was somewhat exaggerated. It was concluded that adding an axial velocity field that is irrotational does not change the errors when the measuring pane is tilted but if there is vorticity associated with the axial-flow distribution, such as with the Gaussian distribution, significant distortions will occur in the $\gamma$ distribution and sizable errors arise in determining the vortex centre location.

Acknowledgements The authors wish to acknowledge the assistance of Scuderia Ferrari Formula 1 who supported this study.

Open Access This article is distributed under the terms of the Creative Commons Attribution 4.0 International License (http://creativeco mmons.org/licenses/by/4.0/), which permits unrestricted use, distribution, and reproduction in any medium, provided you give appropriate credit to the original author(s) and the source, provide a link to the Creative Commons license, and indicate if changes were made.

\section{Appendix}

\section{The Lamb-Oseen vortex}

The distribution of tangential velocity $u_{\theta}$ in an $\mathrm{L}-\mathrm{O}$ vortex is $u_{\theta}=\frac{\Gamma_{0}}{2 \pi r}\left(1-\mathrm{e}^{\frac{-r^{2}}{4 v t}}\right)$,

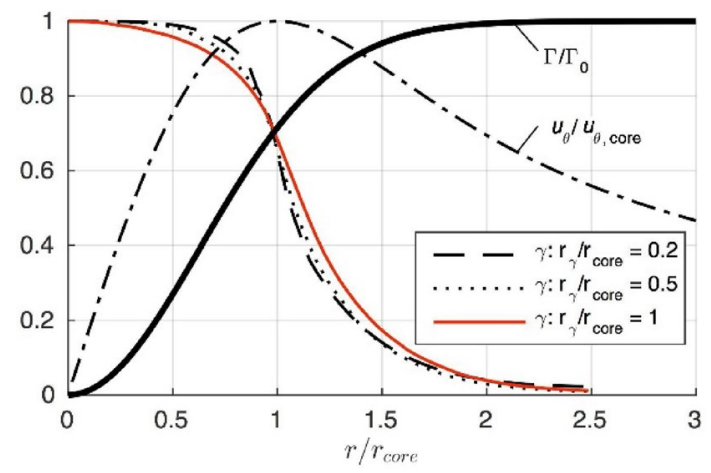

Fig. 14 Distributions of tangential velocity $u_{\theta}$, circulation $\Gamma$ and $\gamma(P)$ for a Lamb-Oseen vortex

where $\Gamma_{0}$ denotes the overall circulation and $4 v t$ represents the viscous diffusion from the core (Lamb 1993). Figure 14 shows the radial distribution of this together with those of the circulation $\Gamma$ and $\gamma(P)$. The latter has been evaluated for three different sized square integration areas $S$, the sides of which $r_{\gamma}$ in length. The vortex core radius $r_{\text {core }}$ is defined as the radius where the swirl velocity reaches its maximum equal to $u_{\theta \text {, core }}$, that is at the radius where $r=r_{\text {core }}=1.121 \sqrt{4 v t}$. The circulation can be evaluated knowing the magnitude of the tangential velocity at this point using the expression

$\boldsymbol{\Gamma}_{0}=\frac{2}{1-e^{-1.25643}} r_{\text {core }} u_{\theta, \text { core }}=8.78 r_{\text {core }} u_{\theta, \text { core }}$

\section{References}

Berdahl CH, Thompson DS (1993) Eduction of swirling structure using the velocity gradient. AIAA J 31:97-103

Bertényi T (2001) Merger of aircraft wake vortices. $\mathrm{PhD}$ thesis, University of Cambridge

Bertényi T, Graham WR (2007) Experimental observations of the merger of co-rotating wake vortices. J Fluid Mech 586:397-422

Chakraborty P, Balanchandar S, Adrian RJ (2005) On the relationship between local vortex identification Schemes. J Fluid Mech 535:189-214

Graftieaux L, Michard M, Grosjean N (2001) Combining PIV, POD and vortex identification algorithms for the study of unsteady turbulent swirling flows. Meas Sci Technol 12:1422-1429

Jeong J, Hussain F (1995) On the identification of a vortex. J Fluid Mech 285:69-94

Jiang M, Machiraju R, Thompson D (2002) A novel approach to vortex core region detection. In: IEEE symposium on visualisation, pp 217-224

Lamb SH (1993) Hydrodynamics. 6th edn. Cambridge University Press, Cambridge

Morgan CE (2010) Unsteady vortex interactions related to a formula one car front wing and wheel, PhD Thesis, University of Cambridge,

Pegrum JM (2006) Experimental study of the vortex system generated by a Formula 1 front wing. Ph. D. thesis, Imperial College, London

Pemberton RJ, Turnock SR, Dodd TJ, Rogers E (2002) A novel method for identifying vortical structures. J Fluid Struct 16:1051-1057 
Schram C, Rambaud P, Riethmuller ML (2004) Wavelet based eddy structure eduction from a backward facing step flow investigated using particle image velocimetry. Exp Fluid 36:233-245

Shah MN (2005) A study of vortex interaction phenomena related to formula one cars. Ph.D. thesis, University of Cambridge

Varun A, Balasubramanian K, Sujith R (2008) An automated vortex detection scheme using the wavelet transform of the $\mathrm{d}_{2}$ field. Exp Fluid 45:857-868

Vollmers H (2001) Detection of vortices and quantitative evaluation of their main parameters from experimental velocity data. Meas Sci Technol 12:1199-1207
Wang L, Hu R, Zhang J, Ma Y (2015) On the vortex detection method using continuous wavelet transform with application to propeller wake analysis. Math Probl Eng 2015:242917

Publisher's Note Springer Nature remains neutral with regard to jurisdictional claims in published maps and institutional affiliations. 